(C) 2004 IEEE. Personal use of this material is permitted. Permission from IEEE must be obtained for all other uses, in any current or future media, including reprinting/republishing this material for advertising or promotional purposes, creating new collective works, for resale or redistribution to servers or lists, or reuse of any copyrighted component of this work in other works. 


\title{
Performance Analysis and Parameter Optimization of DLL and MEDLL in Fading Multipath Environments for Next Generation Navigation Receivers
}

\author{
Matilde Sánchez-Fernández, Member, IEEE, Miguel Aguilera-Forero, Student Member, IEEE, \\ and Ana García-Armada, Member, IEEE
}

\begin{abstract}
In this paper the choice of DLL parameters is studied with special focus on multipath and Doppler sensitivity. The envisaged application is code tracking on navigation receivers and the multipath fading environments defined for next generation navigation systems are considered. Given the particular properties of these propagation environments, multipath estimating delay lock loop is shown to have the best performance within a particular signal to noise ratio range if some specific parameters such as the Predetection Integration Time and channel estimation time interval match the time-varying nature of the channel and the spacing in the early-late scheme is chosen accordingly to the multipath characteristics ${ }^{1}$.
\end{abstract}

Index Terms - Navigation receivers, MEDLL, multipath, slow fading.

\section{INTRODUCTION}

Satellite navigation idiosyncrasy leads to challenging studies in synchronization techniques given that the delay estimation is the key procedure to determine the position of the receiver.

The key issues in synchronization are code acquisition and tracking. From both, code tracking is especially sensitive to multipath environments and the time-varying nature of satellite channels [1]-[3].

Several multipath mitigating techniques have been compared and studied [4]-[7] for code tracking. From Delay Lock Loop (DLL) architectures the most significant and promising tracking scheme to be studied is the multipath estimating DLL (MEDLL), since it has proven best performance in multipath environments [8]-[10]. However, issues such as the Doppler sensitivity of the multipath estimation track and time integration intervals, the signal to noise ratios where the channel estimates are accurate enough to provide a satisfactory performance and the early-late spacing should be particularly addressed.

\footnotetext{
${ }^{1}$ This study has been partly funded by ESA/ESTEC Contract Number 15534/01/NL/LvH.

M. Sánchez-Fernández is with the Department of Signal Theory and Communications, Univ. Carlos III de Madrid, Av. de la Universidad 30, 28911 Leganés, Spain (e-mail: mati@tsc.uc3m.es).

M. Aguilera-Forero is with in the Satellite Navigation Division of AENA, Ctra. de la Base s/n 28850 - Torrejón, Spain (e-mail: maguilera@aena.es).

A. García-Armada is with the Department of Signal Theory and Communications, Univ. Carlos III de Madrid, Av. de la Universidad 30, 28911 Leganés, Spain (e-mail: agarcia@tsc.uc3m.es).
}

Next generation navigation receivers require an even more careful design of synchronization techniques [11]-[13] since it is aiming at a higher positioning accuracy than previous systems. Also, channel models for these new systems are recently available in the literature [14]-[17], thus the performance and parametrization of DLL architectures should be considered under these new environments.

The novelty of our study is to consider the time varying nature of the multipath channel in the particular case of a next generation navigation receiver and to address the optimization of several parameters in order to accomplish the expected performance in code tracking. Under these premises, an accurate choice of the Predetection Integration Time (PIT) interval and the channel estimation time interval in the DLL scheme is shown to be critical. Also the optimal spacing in the early-late scheme is shown to be highly dependent on the multipath characteristic, particularly in these environments where there is a strong specular component and most of the power delay profile is concealed within a small chip time interval.

The remainder of this paper is organized as follows. Section II provides an overview of synchronization schemes and a brief review of multipath propagation. In section III the system model is presented. In section IV the obtained results are discussed and finally section $\mathrm{V}$ outlines some concluding remarks.

\section{SYNCHRONIZATION TECHNIQUES AND MULTIPATH EFFECTS}

\section{A. DLL and MEDLL schemes}

The simplest DLL scheme correlates the received signal $r(t)$ with both an advanced $s_{e}(t)$ and a delayed $s_{l}(t)$ normalized version of the internally generated reference prompt code $s_{0}(t)=s\left(t-\epsilon_{i} T_{c}\right)$ (Fig. 1). This reference code has an inherent delay $\in_{i}$ (normalized to the chip period $T_{c}$ ) with respect to the transmitted code $s(t)$. The suffix $i$ represents each detection interval $T_{p}$ named PIT (Predetection Integration Time). This delay should be estimated and compensated through the synchronization procedure. The shifting time for $s_{e}(t)$ and $s_{l}(t)$ is $\pm \Delta / 2 \times T_{c}$ where $\Delta$ is the correlation spacing (in chip fractions). 


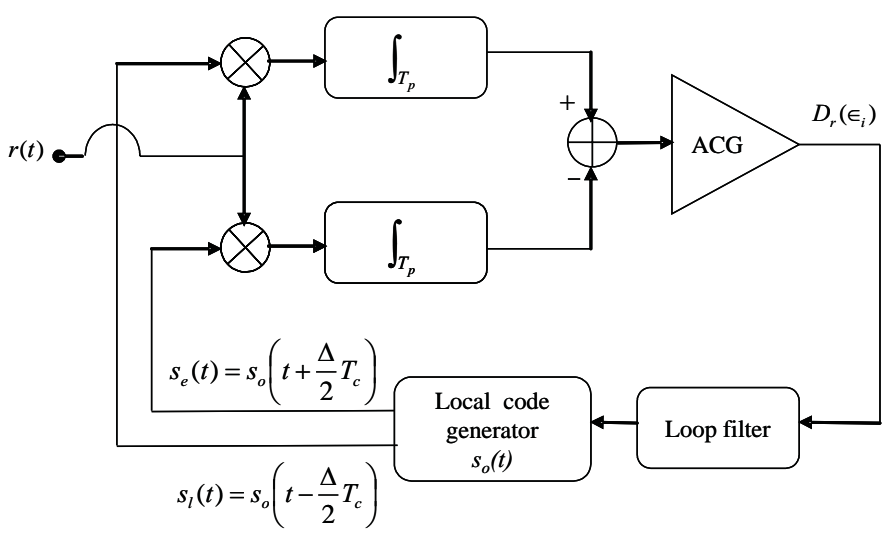

Fig. 1. Block Diagram of an Early-Late DLL.

More practical DLL schemes separate the in-phase and quadrature components of the received base-band signal and correlate each with the early, late and prompt versions of the internally generated code. Different combinations of these correlation outputs lead to coherent and non-coherent DLL discriminators. A coherent discriminator requires that the receiver is phase-locked to the signal. In most positioning applications based on spread spectrum this is unrealistic because of the low signal to noise ratio (SNR) that does not allow carrier recovery before code synchronization [2]. Furthermore, the performance advantage of non-coherent discriminators versus coherent has been previously reported not only in AWGN channels [18] but also in multipath environments [19].

MEDLL is a DLL where the multipath parameters are estimated in order to improve the correlation function of the direct signal [8]-[10]. The estimated correlation function of the reflected rays is subtracted from the correlation function of the received signal to obtain the direct path correlation. Generally speaking, MEDLL reduces the error due to multipath in both, code and carrier tracking. It approaches the ideal performance (no multipath) as the channel estimates improve. However it suffers from higher complexity due to the channel estimation procedure, and high Doppler and SNR sensitivity.

The correlation spacing $\Delta$ in the DLL is chosen as a trade off between tracking accuracy (small values of $\Delta$ ) and the range of delays for which the synchronization may be achieved [20], [18], [2], [5]. Analytical developments show that this still holds for multipath environments. However this general statement may need to be clarified for particular and more realistic multipath fading environments.

Any disturbance or mismatch in the system leads to a estimated delay $\epsilon_{i}^{\prime}$ that may be different from $\epsilon_{i}$. The tracking error $e_{i}=\epsilon_{i}^{\prime}-\epsilon_{i}$, is the difference between both. The performance of a DLL is usually measured in terms of the tracking error bias $\left(E\left\{e_{i}\right\}\right)$ and the tracking error variance ( $\left.E\left\{\left(e_{i}-E\left\{e_{i}\right\}\right)^{2}\right\}\right)$ [3], [21]. This performance is a function of the disturbances, the discriminator chosen and the loop bandwidth $\left(B_{L}\right)$ that in the absence of a loop filter is mainly dependent on the PIT interval.

In the absence of noise or any other disturbance, the detection voltage (discrimination function) $D_{r}\left(\epsilon_{i}\right)$ is a quasi-linear function of the prompt code delay $\epsilon_{i}$ in the interval $\left|\epsilon_{i}\right| \leq \Delta / 2$, thus it can be approximated by:

$D_{r}\left(\epsilon_{i}\right)=2 f(\Delta) \epsilon_{i}$

where $f(\Delta)$ is a linear function of the correlation spacing ${ }^{2}$.

When the input signal and the prompt code are synchronized, $\epsilon_{i}=0$ and consequently $D_{r}\left(\epsilon_{i}\right)=0$. If not, it will be necessary to move along the curve $D_{r}\left(\epsilon_{i}\right)$ to obtain the synchronization condition.

\section{B. Multipath Propagation}

Multipath propagation causes the reception of several replicas of the transmitted signal. In a multipath channel, depending on the nature of the paths, the impulse response can be diffuse or discrete. The channel environments proposed for study in section III-B match a discrete multipath model that assuming a slow fading characteristic behaves as follows:

$c(\tau, t)=\sum_{k=1}^{N} a_{k}(t) \delta\left(t-\tau_{k}\right)$

Multipath $c(\tau, t)$ can be modeled by a wide sense stationary uncorrelated scattering random process (WSSUS). If $c(\tau, t)$ is a zero mean process, then its amplitude $R=|c(\tau, t)|$ can be modeled as a Rayleigh distribution and each of the fading taps are zero-mean complex Gaussian distributions. On the other hand, if $c(\tau, t)$ is a non-zero mean process, then its amplitude $R$ may be represented by a Ricean distribution with Rice factor $K$. Each of the random processes that model the fading taps has a power spectral density parameterized through the Doppler frequency $f_{d}$ :

$f_{d}=\frac{v f_{0}}{c}$

where $c$ is light speed, $v$ is the user's speed and $f_{0}$ is the carrier frequency.

$2 f(\Delta)$ depends mainly of the discriminator chosen. Coherent DLL usually leads to $f(\Delta)=1$ while non-coherent such as the Dot Product [22] leads to $f(\Delta)=(1-\Delta / 2)$. 


\section{SYSTEM MODEL}

\section{A. Signal model and DLL receiver architecture}

The European next generation navigation system provides ten signals within the frequency ranges $1164-1215 \mathrm{MHz}$ (E5a and E5b bands), 1215-1300 MHz (E6 band) and 1559$1592 \mathrm{MHz}$ (E2-L1-E1 bands) [23]. Six of those signals will be available for all users in bands E5a, E5b and L1 for "Open Services" (OS) and "Safety-of-Life Services" (SoL). Two other signals on band E6 will be used for "Commercial Services" (CS) and the remaining two, one in band E6 and another in band E2-L1-E1 will be exclusively accessed for "Public Regulated Service" (PRS) users.

All satellites share the previously mentioned bands using CDMA. Signals from different satellites are distinguished through different codes. The chip rates of the codes range from $R_{c}=1023 \mathrm{Mchip} / \mathrm{s}$ to $R_{c}=10023 \mathrm{Mchip} / \mathrm{s}$ and the modulation schemes used are either BPSK (Binary Phase Shift Keying) or BOC (Binary Offset Carrier) [24].

For our signal model just one signal from one of the satellites is considered whereas the contribution from other satellites is modeled as AWGN due to the weakness of their interference. This is possible since Gold codes with good autocorrelation and cross-correlation properties are being used for spreading the signal. The data signal is dropped in our model since its influence can be removed by envelope detection in the non-coherent DLL that is going to be used as explained latter [1]. The internal code is generated with shift registers of $m=10$ positions, so, considering a Gold sequence, the code length is $N_{c}=2^{m}-1=1023$ chips. The reason for choosing the lowest rate is that this way we get an idea of the lowest performance expected in the system in terms of tracking error. For the sake of simplicity, the modulation used in the model will be BPSK. BOC waveforms have autocorrelation functions with multiple peaks that lead to tracking ambiguities [25], thus the discriminator should deal with this problem by increasing its complexity.

The discriminator used is a non-coherent Dot Product DLL [22]. The correlation spacing ranges from $\Delta=0.2$ to $\Delta=1$ chips. Setting the code length to $N_{c}=1023$, the code period will be $T_{s}=N_{c} \times T_{c}=1 \mathrm{~ms}$. Each detection interval (PIT), identified with the suffix $i$, has a duration of $T_{p}=N_{p} \times T_{s}$ seconds, where $N_{p}$ varies for different loop bandwidth values. The MEDLL implementation tracks for any path within $0.2 \times T_{c}$ seconds. This is a valid approach, since it should be noted that none of the channel delay profiles shown in next section extends further than $0.3 \times T_{c}$ seconds. The estimation of the channel amplitude is performed minimizing the mean square error of the estimates as detailed in [9].
The front-end filter bandwidth is set to be a large enough value ( $\approx 20 \mathrm{MHz}$ ) to avoid distortion of the correlation function and focus on the performance of the DLL in multipath fading [1]. Therefore, the presented results would hold for a sufficiently large bandwidth.

Thus, assuming slow fading multipath, the synchronization base-band signal arriving to the receiver can be expressed as:

$r(t)=\sum_{k=1}^{N} a_{k}(t) s\left(t-\tau_{k}\right)+n(t)$

where $a_{k}(t)$ represent the multipath coefficients from (2), $n(t)$ is white Gaussian noise and $s(t)$ is the transmitted signal with the parameters previously described.

\section{B. Channel models}

The channel models considered for L-band [15] are described in terms of a discrete power-delay profile and the Doppler spectrum (Tables I-III).

TABLE I

EN-ROUTE AERONAUTICAL (E-RA) CHANNEL

\begin{tabular}{cccc}
\hline \hline Delay (ns) & $\begin{array}{c}\text { Relative Power } \\
\text { (dB) }\end{array}$ & $\begin{array}{c}\text { Statistical } \\
\text { distribution }\end{array}$ & $f_{d}$ (Hz) \\
\hline 0 & -18 & Ricean $K=15 \mathrm{~dB}$ & 1 \\
50 & -6 & Gaussian & 1 \\
\hline \multicolumn{4}{c}{ TABLE II } \\
LAND-MobILE IN URBAN (L-MU4 OR L-MU70) CHANNEL \\
\hline \hline Delay (ns) & Relative Power & Statistical & $f_{d}$ (Hz) \\
\multicolumn{5}{c}{ distribution } \\
\hline 0 & -10 & Ricean $K=7$ dB & 4 or 70 \\
60 & -27 & Gaussian & 4 or 70 \\
100 & -27 & Gaussian & 4 or 70 \\
130 & -27 & Gaussian & 4 or 70 \\
250 & -27 & Gaussian & 4 or 70 \\
\hline
\end{tabular}

TABLE III

LAND-MOBILE IN RURAL (L-MR) CHANNEL

\begin{tabular}{cccc}
\hline \hline Delay (ns) & $\begin{array}{c}\text { Relative Power } \\
(\mathbf{d B})\end{array}$ & $\begin{array}{c}\text { Statistical } \\
\text { distribution }\end{array}$ & $f_{d} \mathbf{( H z )}$ \\
\hline 0 & -9 & Ricean $K=6 \mathrm{~dB}$ & 140 \\
100 & -28 & Gaussian & 140 \\
250 & -31 & Gaussian & 140 \\
\hline
\end{tabular}

A tapped delay line is used for implementing the channel models. The channel coefficients $a_{k}(t)$ are obtained by filtering independent samples of a complex white Gaussian random variable with a Butterworth filter designed for a $3 \mathrm{~dB}$ bandwidth equal to the Doppler frequency $f_{d}$.

\section{Discussion AND Simulation Results}

The performance of the DLL architectures will be evaluated considering the different multipath scenarios given in previous section. The tracking error bias and the tracking error variance are obtained through simulation for $C / N_{0}$ values ranging 
from 30 to $60 \mathrm{dBHz}$, different $T_{p}$ and different correlation spacings. The underlying idea in this section is to determine the choice of parameters needed in a DLL architecture to set the tracking error within a range that allows the performance expected in next generation positioning systems [21] ${ }^{3}$.

First a discussion on the necessary channel estimation updates is made for MEDLL in order to set the MEDLL in the best possible channel estimation scenario. Later, MEDLL and DLL performance is studied from two points view: Doppler sensitivity and multipath bias. For both scenarios, a discussion is given on the alternatives to MEDLL in order to avoid the increased complexity that channel estimates lead to.

\section{A. MEDLL and channel estimation}

The Gold sequence period is $1 \mathrm{~ms}$, which implies that this is the smallest granularity we will get in the channel estimates. Compared to other system chip rates this is the worst scenario too. A trade-off between increased complexity in the MEDLL scheme and applying accurate updated channel estimates should be made. It is obvious that within each detection interval $\left(T_{p}\right)$, updates of the channel estimate are necessary every $T_{\text {est }}$ seconds and that this depends on the Doppler frequency or the channel coherence time $T_{\text {coh }}$. How many of these updates are necessary within $T_{p}$ will be discussed here. Another issue to take into account is the low $C / N_{0}$ the receiver has to cope with and that might place the MEDLL in disadvantage compared to other schemes that do not need channel estimates.

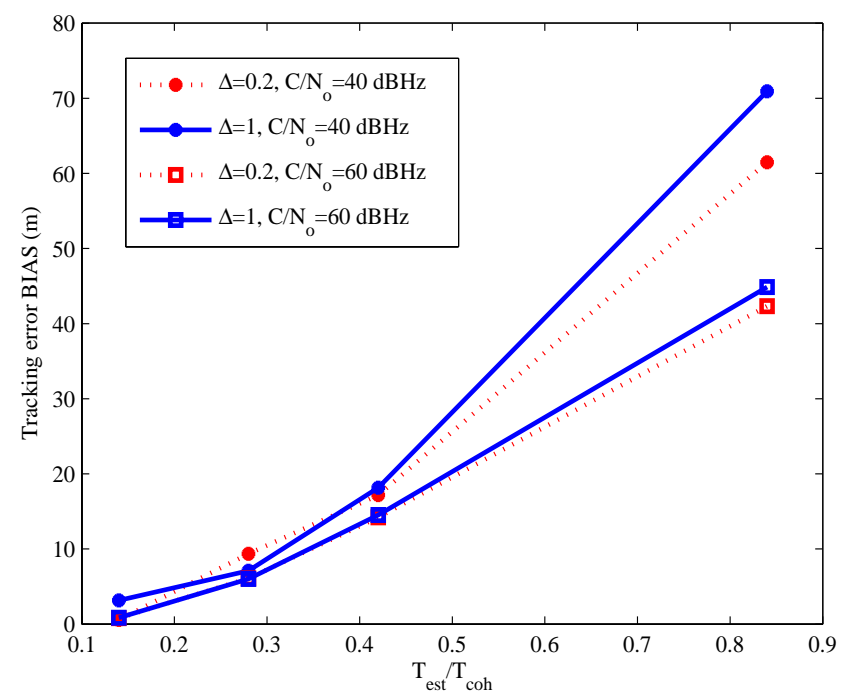

Fig. 2. MEDLL performance for different channel estimation interval ( $T_{\text {est }}$ ) in channel L-MR.

3 Performance is usually specified in terms of different components: Ionosphere, Troposphere, Signal in Space Accuracy (SISA), Noise + Interference, Multipath, etc. In our study we are just focusing in Multipath and Noise + Interference performance.
MEDLL tracking error bias for different channel estimates is shown in figure 2. These results have been obtained for "Land-Mobile in Rural" channel (L-MR): since it has a stronger multipath component, it is a conservative performance bound.

Also, this channel performs similar to "Land-Mobile in Urban environment" channel with $f_{d}=70 \mathrm{~Hz}$ (L-MU70) and "Land-Mobile in Urban environment" with $f_{d}=4 \mathrm{~Hz}$ (LMU4) in terms of multipath. For example, if we want to keep the tracking error bias below a 10m (meter) threshold for low SNR scenarios it should be necessary to estimate every $0.2 \times T_{\text {coh }}$ seconds or less. Assuming that $T_{\text {coh }}=\left(f_{d}\right)^{-1}$, the LMR channel is placed in an scenario where estimates have to be obtained approximately every $1 \mathrm{~ms}$ (the exact value is 1.4 $\mathrm{ms}$ ) and, given that this is the smallest granularity we may achieve, this is independent of the $T_{p}$ that is being used. The L-MU70 channel may update its channel estimates every 3ms approximately. This implies that the number of updates within $T_{p}$ should be chosen accordingly. For channels "En-Route Aeronautical” (E-RA) and L-MU4 channels, the worst case leads to updates every 50ms. Taking into account a reasonable range of $T_{p}$ values, no channel updates would be necessary within $T_{p}$ for these two channels.

It should be noted here that any estimation of the delay value is allowed for these simulations with the purpose of making the figure clearer, despite of the fact that it is known that the delay estimation is valid when the absolute value of the measurement is within $\Delta / 2$ chip time. Consequently, any value greater than $29.3 \mathrm{~m}$ for $\Delta=0.2$ and $146.6 \mathrm{~m}$ for $\Delta=1$ implies that the MEDLL is out-of-track and the receiver should switch to the acquisition procedure. In the rest of the results, any delay estimation that drives MEDLL out-of-track is discarded.

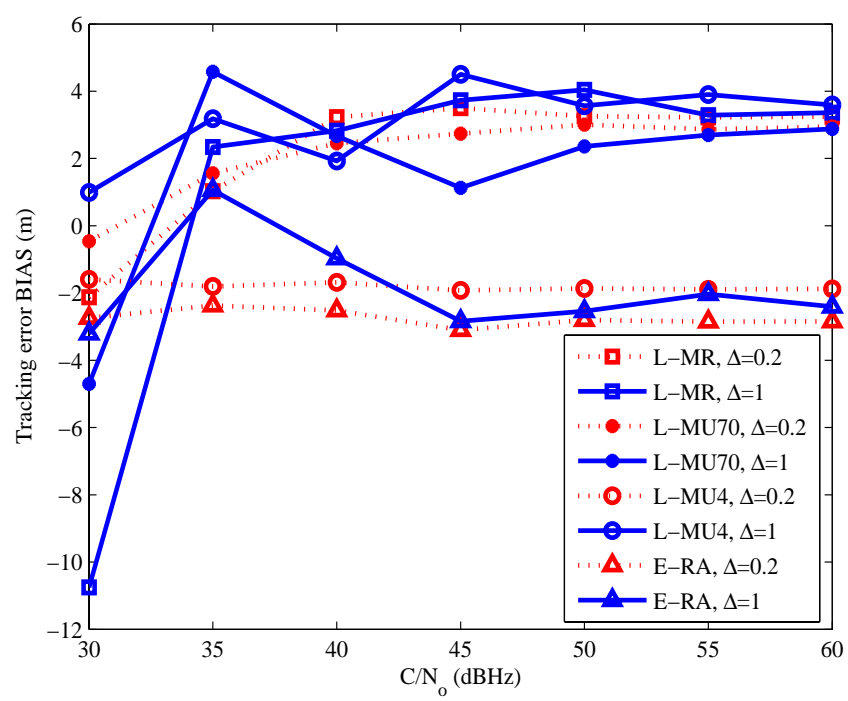

Fig. 3. MEDLL tracking error bias for different $C / N_{0}$ (dBHz). 
In figures 3 and 4 MEDLL behavior for different $C / N_{0}$ values is studied for the four channels scenarios described in section III-B. The results shown are for the best possible channel estimation interval $\left(T_{\text {est }}=1 \mathrm{~ms}\right)$ and for an adverse Doppler scenario where $T_{p}$ is much smaller that $T_{\text {coh }}$ (see next section). The performance for the different scenarios is studied first by determining in each case what is the percentage of time the MEDLL is out-of-track. Then the error bias and variance are obtained taking into account that any out-of-track measure is discarded.

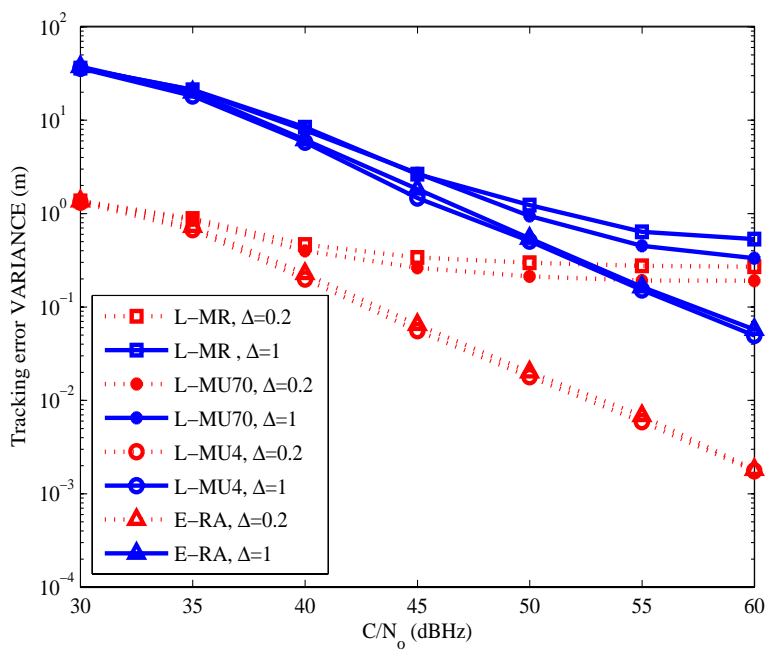

Fig. 4. MEDLL tracking error variance for different $C / N_{0}$ (dBHz).

Table IV shows the percentage of time the MEDLL is outof-track for different $C / N_{0}$ values. In all cases, values of $C / N_{0} \leq 35 \mathrm{dBHz}$ lead to unacceptable performance. Also, as expected, the narrow DLL ( $\Delta=0.2$ ) performs worst in high variability environments.

TABLE IV

PROBABILITY OF MEDLL OUT-OF-TRACK

\begin{tabular}{ccccccc}
\hline \hline$C / N_{0}$ & $\mathbf{3 0}$ & $\mathbf{3 5}$ & $\mathbf{4 5}$ & $\mathbf{4 0}$ & $\mathbf{5 0}$ & $\mathbf{6 0}$ \\
$\mathbf{( d B H z )}$ & $\mathbf{( d B H z )}$ & $\mathbf{( d B H z )}$ & $\mathbf{( d B H z )}$ & $(\mathbf{d B H z})$ & $\mathbf{( d B H z )}$ \\
\hline $\begin{array}{c}\text { E-RA } \\
\Delta=0.2\end{array}$ & $30.8 \%$ & $8.6 \%$ & $0.4 \%$ & $\downarrow 0.1 \%$ & $\downarrow 0.1 \%$ & $\downarrow 0.1 \%$ \\
$\begin{array}{c}\text { E-RA } \\
\Delta=1\end{array}$ & $33.3 \%$ & $10.7 \%$ & $0.3 \%$ & $\downarrow 0.1 \%$ & $\downarrow 0.1 \%$ & $\downarrow 0.1 \%$ \\
$\begin{array}{c}\text { L-MU4 } \\
\Delta=0.2\end{array}$ & $27.5 \%$ & $7 \%$ & $0.1 \%$ & $\downarrow 0.1 \%$ & $\downarrow 0.1 \%$ & $\downarrow 0.1 \%$ \\
$\begin{array}{c}\text { L-MU4 } \\
\Delta=1\end{array}$ & $32.2 \%$ & $8.6 \%$ & $0.4 \%$ & $\downarrow 0.1 \%$ & $\downarrow 0.1 \%$ & $\downarrow 0.1 \%$ \\
$\begin{array}{c}\text { L-MU70 } \\
\Delta=0.2\end{array}$ & $29.8 \%$ & $14 \%$ & $3.8 \%$ & $1.6 \%$ & $0.7 \%$ & $0.6 \%$ \\
$\begin{array}{c}\text { L-MU70 } \\
\Delta=1\end{array}$ & $33.2 \%$ & $14.1 \%$ & $1.6 \%$ & $0.3 \%$ & $\downarrow 0.1 \%$ & $\downarrow 0.1 \%$ \\
$\begin{array}{c}\text { L-MR } \\
\Delta=0.2\end{array}$ & $31.1 \%$ & $14.2 \%$ & $5.9 \%$ & $3.2 \%$ & $2.3 \%$ & $1.8 \%$ \\
$\begin{array}{c}\text { L-MR } \\
\Delta=1\end{array}$ & $33.7 \%$ & $13.6 \%$ & $2.7 \%$ & $0.1 \%$ & $0.2 \%$ & $0.2 \%$ \\
\hline
\end{tabular}

Although from observation of figure 3 it might seem that almost all channels are performing within a $10 \mathrm{~m}$ error threshold, it should be taken into account that this is the bias of the error, and in order to establish the error with a confidence interval its variance should also be taken into account (Figure 4). The narrow MEDLL is in almost all scenarios below an error variance of $1 \mathrm{~m}$, while the conventional MEDLL needs in most cases more than $50 \mathrm{dBHz}$ of $C / N_{0}$ to achieve this value.

\section{B. Tracking error Doppler sensitivity}

Previous results in literature [1] show that for a given channel coherence time $\left(T_{c o h}\right)$ decreasing the loop bandwidth (increasing PIT) reduces the tracking error bias; up to a point where for coherent DLL it could totally disappear ${ }^{4}$. This idea suggests that tuning $T_{p}$ accordingly to the channel coherence time might lead to DLL strategies where MEDLL complexity is not needed, even for non-coherent DLL. Therefore, increasing $T_{p}$ shows up as a strategy for reducing the error bias that at some point might avoid the need of channel estimates. This statement has certain limitations that will be shown with the results.

To discuss on the previous theoretical results, the behavior of DLL and MEDLL strategies is studied for L-MU channel. This particular channel is chosen since it specifies two Doppler frequencies and allows a reasonable range of $T_{p}$ to be studied. The results obtained are for the best possible channel estimation interval $\left(T_{\text {est }}=1 \mathrm{~ms}\right)$ and for two different values of $C / N_{0}=\{40,60\} \mathrm{dBHz}$. The performance for the different scenarios is studied first by determining in each case what is the percentage of time the DLL or MEDLL is out-oftrack. Then the error bias and variance are measured taking into account that again, any measure out-of-track is discarded.

TABLE V

\begin{tabular}{ccccc}
\hline \hline$T_{p} / T_{\text {coh }}, C / N_{0}$ & DLL & DLL & MEDLL & MEDLL \\
& $\Delta=0.2$ & $\Delta=1$ & $\Delta=0.2$ & $\Delta=1$ \\
\hline $0.07,40 \mathrm{dBHz}$ & $47.3 \%$ & $3.75 \%$ & $4.65 \%$ & $1.8 \%$ \\
$0.07,60 \mathrm{dBHz}$ & $46.6 \%$ & $1.8 \%$ & $1 \%$ & $\downarrow 1 \%$ \\
$0.35,40 \mathrm{dBHz}$ & $47.3 \%$ & $1 \%$ & $1.25 \%$ & $2 \%$ \\
$0.35,60 \mathrm{dBHz}$ & $46.6 \%$ & $1 \%$ & $\downarrow 1 \%$ & $\downarrow 1 \%$ \\
$0.70,40 \mathrm{dBHz}$ & $29.5 \%$ & $0.5 \%$ & $\downarrow 1 \%$ & $2 \%$ \\
$0.70,60 \mathrm{dBHz}$ & $29 \%$ & $\downarrow 1 \%$ & $\downarrow 1 \%$ & $\downarrow 1 \%$ \\
$1.05,40 \mathrm{dBHz}$ & $26 \%$ & $\downarrow 1 \%$ & $2 \%$ & $1.3 \%$ \\
$1.05,60 \mathrm{dBHz}$ & $27 \%$ & $\downarrow 1 \%$ & $\downarrow 1 \%$ & $\downarrow 1 \%$ \\
\hline
\end{tabular}

Table $\mathrm{V}$ shows the percentage of time the DLL and MEDLL are out-of-track for different $T_{p} / T_{\text {coh }}$ and $C / N_{0}$ values. Although, theoretically, narrow DLL may be an

\footnotetext{
${ }^{4}$ This is not generally true for the case for a non-coherent DLL, however the mentioned behavior is sustained and the error bias still decreases.
} 
alternative to MEDLL strategies in order to solve the error bias problem, from table $\mathrm{V}$ and figures 5 and 6 another conclusion can be drawn.

It can be said that although the narrow DLL $(\Delta=0.2)$ error variance is around $2 \mathrm{~m}$ for any case and its error bias is in the range of $4 \mathrm{~m}$, it is most of the times out-of-track. DLL $(\Delta=1)$ performs better in terms of out-of-track time percentage, but its error bias and variance are considerably higher: error bias is over $8 \mathrm{~m}$ and the variance is around $10 \mathrm{~m}$.

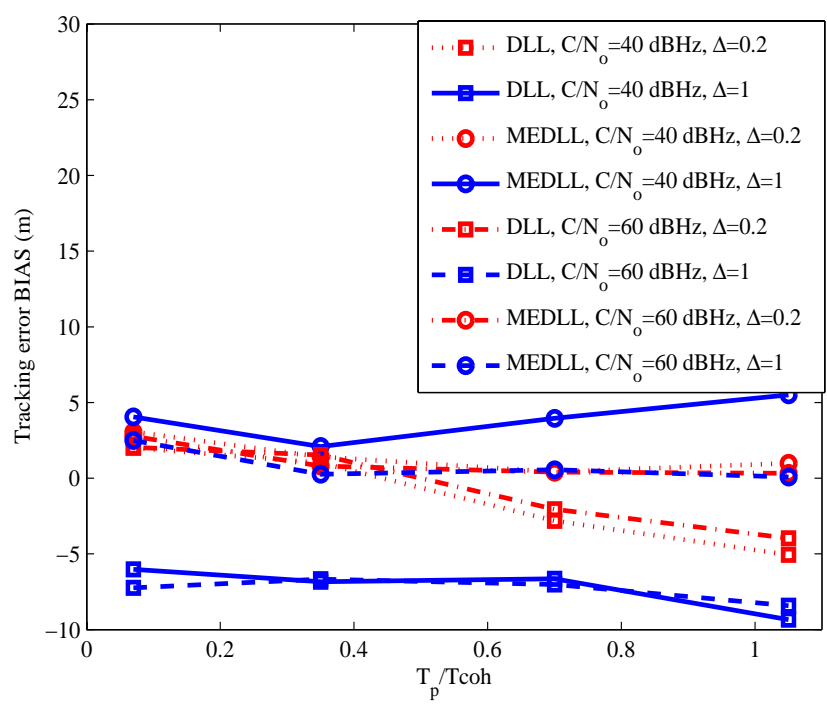

Fig. 5. Tracking error bias for different $T_{p} / T_{c o h}$ in channel L-MU.

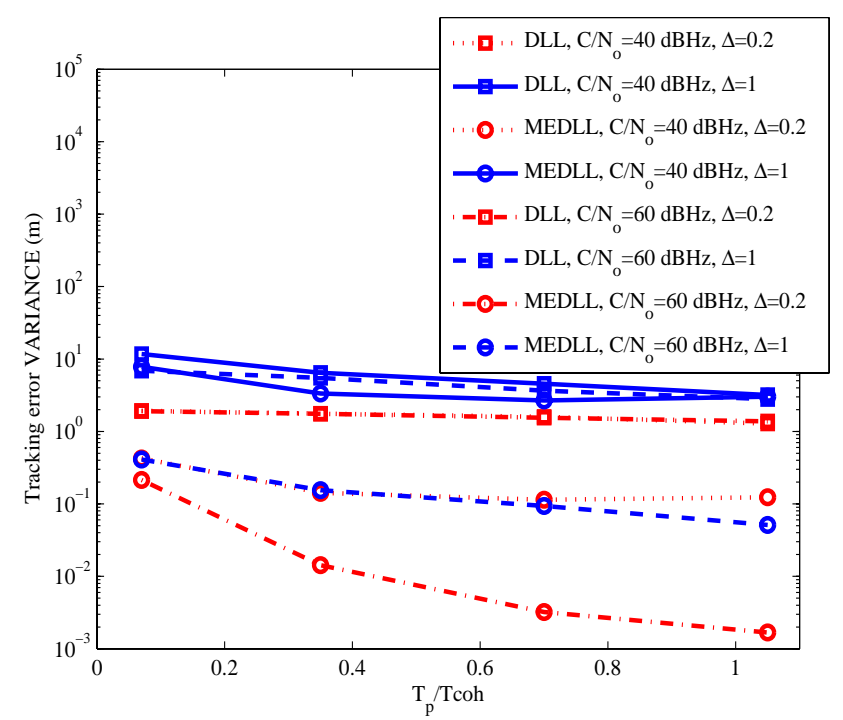

Fig. 6. Tracking error variance for different $T_{p} / T_{c o h}$ in channel L-MU.

MEDLL performance is $2 \mathrm{~m}$ for the error bias, it shows error variance reduction with $T_{p} / T_{c o h}$ and the out-of-track times are negligible. Thus, despite the expected improvement with increasing $T_{p}$ which would make the narrow DLL an alternative in this scenarios, MEDLL still performs better.

\section{Matching multipath parameters and early-late correlation spacing}

The four environments present different multipath scenarios. E-RA channel is the most problematic since it has the strongest multipath component. For strong multipath scenarios it is recommended to increase the correlation spacing, and this is considered in figure 7. As expected, for channel E-RA and L-MU4 there is a slight improvement in the error bias, however, although it is not shown here, this goes together with a worst performance in terms of error variance. In these results $T_{p}=10 \mathrm{~ms}$, a channel estimate is updated every $1 \mathrm{~ms}$ and $C / N_{0}=40 \mathrm{dBHz}$.

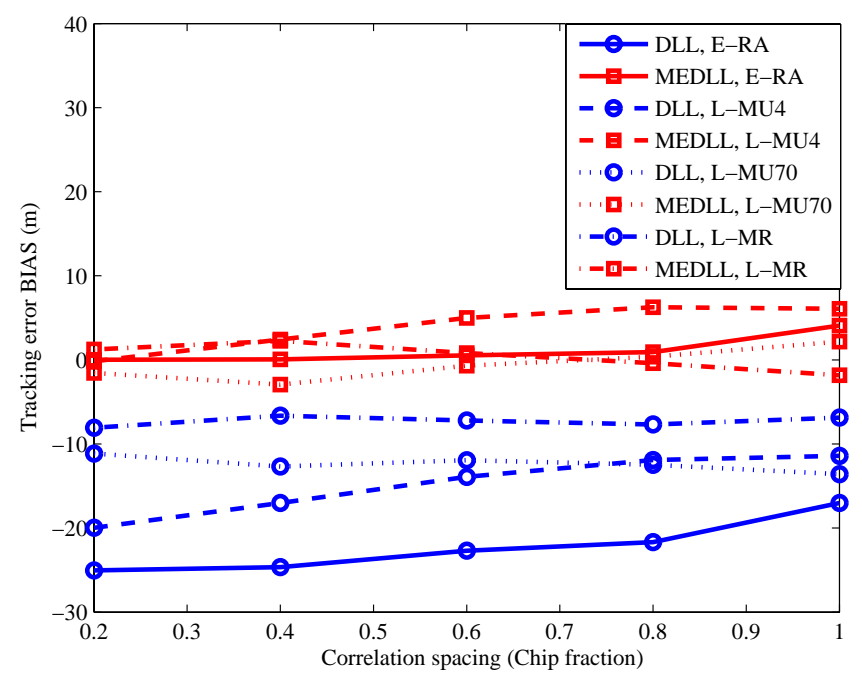

Fig. 7. Tracking error bias for different correlation spacings and different channel scenarios, $C / N_{0}=40 \mathrm{dBHz}$.

\section{CONCLUSION}

The time varying nature of the multipath channel for a next generation navigation environment has been addressed and the values of several parameters have been discussed in order to accomplish a good performance in code tracking.

A detailed study has been made on MEDLL performance, taking into account issues such as channel estimation accuracy for operational SNR values and channel estimation updates for different channel coherence times. Several considerations have been given for error bias and variance performance and the out-of-track performance is provided for the particular scenarios presented.

As an alternative to MEDLL scenarios, and in order to reduce complexity, some other issues have been addressed. Those have been $T_{p}$ increase and $\Delta$ spacing in DLL architectures, in order to scrutinize whether the performance of these schemes was good enough for the proposed scenarios. It has been shown, that although the narrow DLL performs within a reasonable range in error variance and bias, its out-of-track probability is extremely high, which discards this architecture as a possible substitute to MEDLL. 


\section{REFERENCES}

[1] R. V. Nee, "Spread-spectrum code and carrier synchronization errors caused by multipath and interference," IEEE Transactions on Aerospace and Electronic Systems, vol. 29, 1993.

[2] W.-H. Sheen and G. Stuber, "Effects of multipath fading on delaylocked loops for spread spectrum systems," IEEE Transactions on Communications, vol. 42, pp. 1947 - 1956, February-March-April 1994.

[3] J. Soubielle, I. Fijalkow, P. Duvaut, and A. Bibaut, "GPS positioning in a multipath environment," IEEE Transactions on Signal Processing, vol. 50, pp. 141 - 150, January 2002.

[4] M. Braasch and K. van Dierendonck, "GPS receiver architectures and measurements," Proceedings of the IEEE, vol. 87, pp. 48 - 64, January 1999.

[5] M. Braasch, "Performance comparison of multipath mitigating receiver architectures,” in IEEE Aerospace Conference, vol. 3, pp. 1309-1315, March 2001.

[6] G. Heinrichs, R. Bischoff, and T. Hesse, "Receiver architecture synergies between future GPS/Galileo and UMTS/IMT- 2000,” in 56th IEEE Vehicular Technology Conference (VTC 2002-Fall), vol. 3, 2002.

[7] F. Dovis, M. Pini, and P. Mulassano, "Multiple DLL architecture for multipath recovery in navigation receivers," in $59^{\text {th }}$ IEEE Vehicular Technology Conference (VTC 2004-Spring), vol. 5, 2004.

[8] R. V. Nee, "The multipath estimating delay lock loop," in IEEE Second International Symposium on Spread Spectrum Techniques and Applications (ISSTA 92), 1992.

[9] R. V. Nee, J. Siereveld, P. Fenton, and B. Townsend, "The multipath estimating delay lock loop: approaching theoretical accuracy limits,” in IEEE Position Location and Navigation Symposium (PLANS 94), 1994.

[10] B. Townsend, R. V. Nee, P. Fenton, and K. van Dierendonck, "Performance evaluation of the multipath estimating delay lock loop," in ION GPS-95, 1995.

[11] E. Pajala, E. Lohan, T. Huovinen, and M. Renfors, "Enhanced differential correlation method for the acquisition of Galileo signals," in 10th IEEE Singapore International Conference on Communication Systems, 2006.

[12] E. Lohan, "Filter-bank based technique for fast acquisition of Galileo and GPS signals," in EEE 17th International Symposium on Personal, Indoor and Mobile Radio Communications, 2006.

[13] C. Lee, S. Yoo, S. Yoon, and S. Y. Kim, "A novel multipath mitigation scheme based on slope differential of correlator output for Galileo systems," in The 8th International Conference Advanced Communication Technology, vol. 2, 2006.

[14] R. Schweikert and T. Woerz, "Signal design and transmission performance study for GNSS-2,” Final Report, European Space Agency, 1998.

[15] European Space Agency, "Safety of life services ICD." ID/GAL/0080/GLI, Issue 1, 2001.

[16] A. Lehner, A. Steingass, F. Pérez-Fontán, E. Kubista, M. Martín, and B. Arbesser-Rastburg, "Measurement and modeling of the aeronautical channel for Galileo," in 8th European Navigation Conference GNSS, 2004.

[17] E. K. F. Pérez-Fontán B. Sanmartín, A. Steingass and B. ArbesserRastburg, "Measurements and modeling of the satellite to- indoor channel for Galileo," in 8th European Navigation Conference GNSS, 2004.

[18] K. van Dierendonck, P. Fenton, and T. Ford, "Theory and performance of narrow correlator spacing in a GPS receiver," Journal of the Institute of Navigation, vol. 39, pp. 265-283, October 1992.

[19] W.-H. Sheen and C.-H. Tai, “A noncoherent tracking loop with diversity and multipath interference cancellation for direct sequence spreadspectrum systems," IEEE Transactions on Communications, vol. 46, pp. 1516 - 1524, November 1998.
[20] R. V. Nee, "Reducing multipath tracking errors in spread-spectrum ranging systems,” Electronics Letters, vol. 28, pp. 729 - 731, April 1992.

[21] M. Hollreiser, “Galileo receivers - challenges and performance,” in 34th European Microwave Conference, vol. 1, 2004.

[22] T. Felhauer, "Comparison of EML and DOT discriminator DLL multipath performance in GPS/GLONASS navigation receivers," Electronics Letters, vol. 33, pp. 179 - 181, January 1997.

[23] European Space Agency, "Galileo open service signal in space interface control document.” GAL OS SIS ICD/D.0, May, 2006.

[24] N. Martin, V. Leblond, G. Guillotel, and V. Heiries, "BOC(x,y) signal acquisition techniques and performances," in ION GPS/GNSS 2003, 2003.

[25] F. Nunes, E. Sousa, and J. Leitao, "Innovations-based code discriminator for GPS/Galileo BOC signals,” in 60th IEEE Vehicular Technology Conference (VTC2004-Fall), vol. 6, 2004.

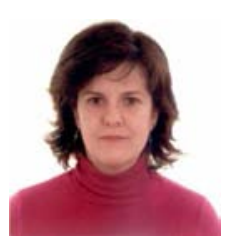

Matilde Sánchez-Fernández (S’99-AM’02-M’04) got her Telecommunications Engineer degree and her PhD from Polytechnic Univ. of Madrid in 1996 and 2001 respectively. She is an Assistant Professor at University Carlos III of Madrid, Spain since April 2000. Previously, she worked for Telefónica as a Telecommunication Engineer. She has performed several research stays at the Information and Telecommunication Technology Center in Kansas University, Bell-Labs, New Jersey and Centre Tecnològic de Telecomunicacions de Catalunya, Spain. She is co-author of two books on wireless communications and has publications on international journals and conferences in this same field. Her current research interests are MIMO techniques, Turbo Codes, mobile communications and simulation and modeling of communication systems.

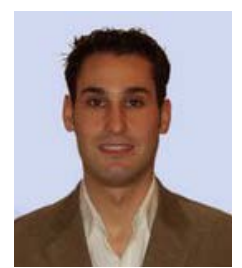

Miguel Aguilera-Forero received the M.Sc degree in Electrical Engineering from the University Carlos III of Madrid in 2003 and is pursuing a PhD in Multimedia and Communications from Universities Carlos III and Rey Juan Carlos. Since 2006, he is with the Division of Satellite Navigation of Aena as a navigation systems engineer (mainly Galileo and EGNOS). His research interests lie in the fields of signal processing for indoor navigation and multipath mitigation.

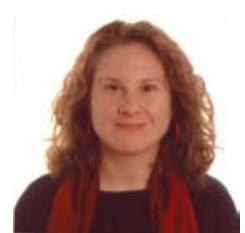

Ana García-Armada (S'95-AM'98-M’00) received the Telecommunication Engineer degree from the Polytechnic University of Madrid (Spain) in July 1994 and Ph.D. in Electrical Engineering from the Polytechnic University of Madrid (Spain), February 1998. She is currently working as an Associate Professor at the University Carlos III de Madrid, Spain where she has occupied several management positions. She has participated in several national and international research projects, most of them related to wireless systems. She is co-author of four books on wireless communications and signal processing. She has published 17 papers in international journals and more than 40 papers in international conferences. She has contributed to international organizations such as ITU and ETSI. She has performed research stays in ESA-ESTEC, Kansas University, Stanford University and Bell Labs. Her research interests are simulation of communication systems, multicarrier and MIMO techniques. 